\title{
Diabetische Fußulzera: Risikofaktor schlechte Haut
}

\begin{abstract}
Strukturelle Hautveränderungen bei Patienten mit neuropathischem Fuß sind ein zusätzlicher Risikofaktor für Fußulzerationen. Zu diesem Schluss kommt eine Studie von Wissenschaftlern der Universität in Birmingham.
\end{abstract}

$E_{\mathrm{b}}^{\mathrm{i}}$ iner Kohorte aus 46 Patienten mit Diabetes und 14 Patienten ohne Diabetes (nicht-diabetische Kontrollen) wurden Hautbiopsien aus Ober- und Unterschenkel entnommen. Von den 46 Diabetikern hatten neun keine Neuropathie, 16 eine periphere diabetische Polyneuropathie und 21 Patienten rezidivierende neuropathische Fußulzera. Keiner der Patienten litt an einer pAVK. Aus den Biopsien wurden die intra-epidermale Nervenfaserdichte (IENFD), Strukturanalysen der Haut, die Mengen an Typ-1-Prokollagen, die gewebezerstörenden Matrix-Metalloproteinasen (MMPs) und die poly (ADPribose) immunereactivity (PAR) bestimmt.

Die IENFD war bei allen Patienten mit diabetischer Polyneuropathie vermindert und es gab keine Unterschiede zwischen den verschiedenen Gruppen mit Neuro- pathien. Die Strukturanalysen der Haut zeigten verminderte Scores bei allen $\mathrm{Pa}$ tienten mit Neuropathie, vor allem bei denjenigen mit Fußulzerationen. Typ1-Prokollagen war bei Patienten mit diabetischen Fußulzerationen signifikant reduziert im Vergleich zu den Kontrollen. Die MMPs waren bei allen Diabetikern aktiviert. Die PAR lag bei Patienten mit Ulzerationen höher als bei Patienten mit Polyneuropathie.

Fazit: Patienten mit neuropathischen Fußkomplikationen weisen sowohl strukturelle Hautveränderungen als auch ein erhöhtes PAR und ein vermindertes Typ1-Prokollagen in der intakten Ober- und Unterschenkelhaut auf. Die Annahme, dass Ulzerationen nur durch die Gefühllosigkeit des Fußes in Verbindung mit lo-

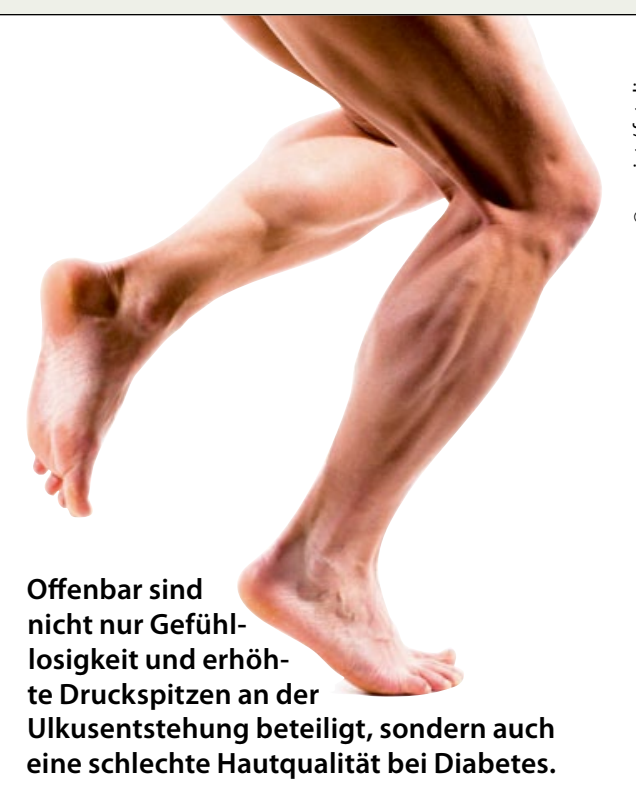

kaler Druckbelastung entstehen, muss um den Faktor einer verminderten Hautqualität beim neuropathischen Fuß ergänzt werden. Prof.Dr.Maximilian Spraul

Tahrani AA et al. Cutaneous structural and biochemical correlates of foot complications in high-risk diabetes. Diabetes Care 2012; 35: 1913-18

\section{Omalizumab wirkt bei idiopathischer Urtikaria}

\begin{abstract}
Mehrere Phase-II-Studien haben bereits gezeigt, dass Omalizumab - ein rekombinanter monoklonaler Antikörper gegen lgE - bei Patienten mit chronischer idiopathischer Urtikaria Juckreiz und Hautsymptome lindern kann. Nun wurden die Ergebnisse der ersten Phase-III-Studie veröffentlicht.
\end{abstract}

$\mathrm{D}$ er Antikörper Omalizumab, der an menschliches IgE bindet, ist in Deutschland zur Add-on-Therapie des allergischen Asthmas zugelassen. Er soll auch bei Patienten mit idiopathischer Urtikaria, bei denen eine Therapie mit H1Antihistaminika wirkungslos bleibt, effektiv sein. Kürzlich wurden Ergebnisse der ersten Phase-III-Studie vorgestellt, in der Prof. Dr. Marcus Maurer, Charité Berlin, und seine Kollegen an 323 Patienten randomisiert und doppelblind die Wirksamkeit und Sicherheit dreier Omalizumab-Dosierungen im Vergleich zu Placebo überprüft haben.

Die Patienten, die auf H1-Antihistaminika nicht angesprochen hatten und zwischen zwölf und 75 Jahre alt waren, bekamen dreimal in jeweils vierwöchigem $\mathrm{Ab}$ stand $75 \mathrm{mg}, 150 \mathrm{mg}, 300 \mathrm{mg}$ Omali- zumab oder Placebo subkutan injiziert und wurden anschließend 16 Wochen nachbeobachtet. Während der gesamten Untersuchungsphase dokumentierten die Patienten morgens und abends den Juckreiz, die Zahl und das Ausmaß der Ausschläge sowie das Auftreten von Angioödemen und deren Therapie.

Nach zwölf Wochen hatte sich der Juckreiz bei Patienten mit $150 \mathrm{mg}$ und 300 mg Omalizumab im Vergleich zur Placebogruppe deutlich gebessert. Die Patienten mit 150 mg Omalizumab stuften ihren Juckreiz im Durchschnitt um 8,1 Punkte $(\mathrm{p}=0,001)$ und die mit $300 \mathrm{mg}$ Omalizumab um 9,8 Punkte ( $<<0,001)$ niedriger ein.

Auch bei weiteren Endpunkten (z.B. Ausmaß der Ausschläge, Veränderungen im Juckreizscore) zeigte sich ein signifi- kanter Unterschied zwischen den Gruppen mit 150-mg-, 300-mg-Dosierung und Placebo, und zwar dosisabhängig.

Über Nebenwirkungen klagten in jeder Gruppe ähnlich viele Patienten: $61 \%$ (Placebo), 59 \% (75 mg Omalizumab), $67 \%$ (150 mg) und $65 \%$ (300 mg). Schwere Nebenwirkungen traten bei neun Fällen auf, die meisten (fünf) entfielen auf die 300-mg-Gruppe.

Fazit: Mit Omalizumab in einer Dosierung von $150 \mathrm{mg}$ und $300 \mathrm{mg}$ lassen sich bei Patienten, die auf H1-Antihistaminika nicht ansprechen, die klinischen Symptome einer chronischen idiopathischen Urtikaria deutlich mildern. Für eine abschließende Beurteilung der Sicherheit sei das Patientenkollektiv jedoch zu klein, resümieren die Autoren, und verweisen auf den großen Anteil schwerer Nebenwirkungen unter der höchsten Dosierung. Dr.Dagmar Kraus

Maurer M et al. Omalizumab for the treatment of chronic idiopathic or spontaneous urticaria. N Engl J Med 2013; 368: 924-35 